\title{
Requirements for Cryopreservation of In Vitro-Produced Bovine Embryos by a Standard Method of Vitrification
}

\section{Do $\mathrm{VH}^{1,2}$, Walton $\mathrm{S}^{3}$, Catt $\mathrm{S}^{4}$ and Taylor-Robinson $\mathrm{AW}^{* 1}$}

${ }^{1}$ School of Medical \& Applied Sciences, Central Queensland University, Rockhampton, QLD, Australia

${ }^{2}$ National Key Laboratory of Animal Cell Technology, National Institute of Animal Sciences, Hanoi, Vietnam

${ }^{3}$ Australian Reproductive Technologies, Mt Chalmers, QLD, Australia

${ }^{4}$ Education Program in Reproduction \& Development, Monash Medical Centre, Clayton, VIC, Australia

${ }^{*}$ Corresponding author: Taylor-Robinson AW, School of Medical \& Applied Sciences, Central Queensland University, Bruce Highway, Rockhampton, QLD 4702, Australia, Tel: 61749232008, E-mail: a.taylorrobinson@cqu.edu.au

Citation: Do VH, Walton S, Catt S, Taylor-Robinson AW (2016) Requirements for Cryopreservation of In Vitro-Produced Bovine Embryos by a Standard Method of Vitrification. J Vet Sci Anim Husb 4(1): 102 doi: 10.15744/2348-9790.4.102

\section{Received Date: August 25, 2015 Accepted Date: January 14, 2016 Published Date: January 15, 2016}

\begin{abstract}
In relation to assisted mammalian reproduction, the goal of cryopreservation is to preserve without significant loss of viability a stock of gametes and/or embryos with a view to thawing those cells for use in in vitro reproduction treatments. There are numerous cryopreservation protocols, which vary in terms of cryoprotectant used, storage temperature, freezing and thawing rates, and the particular cells that they are suitable for preserving. Although slow freezing has become a standard method for in vivo bovine embryo cryopreservation, it seems not to be efficient for preserving in vitro-produced (IVP) bovine embryos. Over the past decade, vitrification, a process of glass-like solidification, has become the cryopreservation method of choice for human oocytes and embryos. This is because it is often less time-consuming than slow freezing, does not require expensive 'slow rate' freezing machines, and has been proven to increase survival rates compared to slow frozen embryos. In contrast to clinical applications, in the cattle industry vitrification presents shortcomings, especially when applied to IVP embryos. A high concentration of cryoprotectant in solution is needed to induce vitrification. However, if left too long with metabolizing embryos, cryoprotectants can become toxic. Failure to standardize vitrification protocols leads to inconsistent results between laboratories, making application less practical in field settings. Therefore, determination of the most suitable vitrification method is important to advance its routine commercial use. Moreover, simplification of the vitrification procedure through development of an in-straw dilution without the use of a microscope may help to expand the use of vitrification methods on the farm.
\end{abstract}

Keywords: Cryopreservation; Slow freezing; Vitrification; Cryoprotectant; Bovine

\section{Introduction}

According to Leibo [1], cryopreservation is an essential component of the embryo transfer industry in cattle that, as Hasler points out [2], is now an established commerce on a global scale. The standard method of bovine embryo cryopreservation, slow freezing, was refined over a period of 40 years of research whereas the alternative vitrification procedure has developed more recently and more rapidly [2].

Pregnancy rates of recipients implanted with in vivo frozen embryos are not inferior to those of recipients receiving fresh embryos $[3,4]$. Nevertheless, several studies have highlighted the inefficiency of this conventional method of cryopreserving in vitro-produced (IVP) bovine embryos [4-9]. These possibly contain more lipid droplets in their cytoplasm than do their in vivo counterparts [10-12], leading to their greater susceptibility to the freezing process $[13,14]$.

In vitro production of bovine embryos has progressed rapidly and they are now relatively cheap to generate in large quantity [4]. Although oocyte numbers from different donors are variable, the in vivo ovum pick-up method coupled with in vitro fertilization and culture can produce an average of 50 calves from repeated aspiration of oocytes of a single donor cow per year [15]. While the yield of IVP embryos may be sizeable, a corresponding number of recipients are often not available [16]. Thus, valuable unused embryos are frequently discarded in the laboratory. Since vitrification is now the main cryopreservation method to store human oocytes and embryos [17], this procedure has also been recommended to assist in the provision of stocks of IVP bovine embryos $[2,4,18]$.

Vitrification is technically simple [19], and does not require a programmable freezer [2,20]. Laboratory experiments have shown that vitrified IVP bovine embryos achieve a better survival rate than do those cryopreserved by slow freezing [4,9,21,22]. As a proof of principle, calves were born successfully after transfer to recipients of vitrified IVP embryos [23,24]; moreover, the pregnancy rates of recipients that are implanted with vitrified IVP embryos may be acceptable in commercial conditions [25]. 
Despite these successful reports, the cattle industry still relies heavily on the transfer of fresh IVP embryos due to the lack of a repeatable cryopreservation technique [16]. There is a plethora of vitrification protocols utilizing different timings, temperatures and cryoprotectants (CPAs). In addition, the user has a choice of vitrification carriers (such as plastic straws, electron microscope grid microdrop, open pulled straw, cryotop, cryohook), with drop-size and straw barriers affecting cooling and warming rates. The warming protocols can differ too, which compounds further the choice of an efficient vitrification method [17]. This is problematic when there is an exchange of vitrified samples between laboratories that adopt different protocols.

Arguably, however, the most important factor to consider in developing a highly successful cryopreservation method is the skill of the operator. Although described as a simple method, without need for slow-rate freezers, in our experience vitrification actually requires more technical dexterity than does slow-freezing.

In parallel with efforts to develop a novel vitrification method, considerable attempts aimed to improve the quality of IVP embryos before cryopreservation have been made. These include modification of in vitro culture systems [12,25], enhancing the intrinsic capacity of embryos [26] and other means of pretreatment [27]. Nevertheless, a significant improvement has so far proved elusive.

This review considers several contributing factors to cryopreservation; ice crystal formation, cooling/warming rates, and the role of CPAs in protecting cells from the chilling process. Moreover, the specific challenges of storing in vitro-fertilized embryos by either slow freezing or vitrification are examined. In addition, attempts to increase the efficiency of vitrification through modifications to in vitro culture and enhancement in the capacity of IVP embryos are discussed. Furthermore, biosafety considerations relevant to vitrification are also taken into account.

\section{Ice Crystal Formation}

During cooling, ice crystals that form outside and inside cells have a detrimental effect on the survival of cryopreserved cells. Seki et al. [28] note that ice crystals occur initially externally during the slow freezing process, a phenomenon to which they refer as extracellular ice crystal formation. According to Jin et al. [29], extracellular ice crystals are harmless to cells, but Mazur et al. [30] maintain that external ice causes distortion to mouse oocytes and their zona pellucida. In addition, intracellular ice formation is one of the most important factors affecting survival of cryopreserved cells [30,31]. Ice crystals forming during the cooling and warming processes are the main cause of cell damage and death [32,33].

Hence, a key aim of cryopreservation is to minimize or even eliminate intracellular ice formation. Kleinhans and Mazur [34] point out that during slow freezing, cell survival is dependent on the cooling rate being sufficiently slow for the volume of unfrozen intracellular water to dehydrate by osmosis to near the equilibrium level before the temperature at which intracellular nucleation occurs is reached. In contrast, Kim et al. [35] and Lawson et al. [36] contend that vitrification eliminates totally intracellular ice crystals. Interestingly, Kobayashi and Kirschvink [37] propose that exposure of cells to electromagnetism reduces ice crystal formation.

\section{Roles of Cooling Rates}

In slow rate cooling, embryos are cooled at a rate of around $0.5^{\circ} \mathrm{C}$ per minute to $-30{ }^{\circ} \mathrm{C}$ or $-35^{\circ} \mathrm{C}$ and are then plunged into liquid nitrogen [1]. In contrast, for vitrification the cooling rates are thousands of degrees Celsius so that each sample containing embryos reaches a glassy state very rapidly. It is thought that two essential elements that vitrification exhibits are high cooling rates and high concentration of cryoprotective additives [28].

Minimum volume vitrification theory relates to the rate of cooling and vitrification carriers. Plastic straws were originally used as the main carrier to vitrify and store oocytes and embryos, but produced poor survival rates of embryos [6]. For example, Palasz et al. [38] achieved around 20\% survival in all experiments in which plastic straws were used. Therefore, cryobiologists have sought to develop a carrier that contains minimum vitrification solution to increase the cooling rates. Kuwayama [39] noted that minimum volume vitrification avoids breakage of zona pellucida, which often occurs when embryos are vitrified in plastic straws. Moreover, the direct contact of vitrification solution with embryos induces ultra-rapid cooling [40]. Vajta et al. [23] reported on a new vitrification carrier, the open-pulled straw (OPS), a $2.5 \mathrm{ml}$ standard plastic straw (used normally for slow freezing embryos) heatsoftened and pulled to decrease the diameter of the straw so that, based on capillary effect, 1-2 $\mu$ l vitrification solution containing oocytes or embryos is spontaneously loaded into the straw. Using the OPS method cooling rates of $25,000{ }^{\circ} \mathrm{C} / \mathrm{min}$ are measured at temperatures from $-25^{\circ} \mathrm{C}$ to $-175^{\circ} \mathrm{C}$, whereas those with the larger plastic straw $(5 \mu \mathrm{l}$, typically used for sperm freezing) are 2,250 ${ }^{\circ} \mathrm{C} / \mathrm{min}$, over ten-fold less [23].

Kuwayama [39] described a specially designed carrier, named Cryotop ${ }^{\oplus}$, comprising a hard plastic holder attached to a narrow and thin film strip, that enables a relatively small volume of vitrification solution containing the sample to be loaded ( $<0.1 \mu \mathrm{l})$. As a result, this device can achieve a cooling rate of $69,250{ }^{\circ} \mathrm{C} / \mathrm{min}$ [28]. However, Rios et al. [19] argue that despite a belief that minimum volume vitrification enhances thermal conductivity and cooling rates, this does not improve the hatching rates of embryos after vitrifying/warming and following in vitro culture. Moreover, Paredes and Mazur [41] noted that vitrification appears not to be influenced by the size of droplets used with a Cryotop ${ }^{\oplus}$. 


\section{Roles of Warming Rates}

Recent research has paid attention to the importance of warming rates because recrystallization affects vitrification outcomes [42]. A process of recrystallization occurs during the warming process [32], during which devitrification can cause cryo-injuries to the cell [43]. Sansinena et al. [43] found that recrystallization starts at $-109^{\circ} \mathrm{C}$. In addition, Hopkins et al. [44] note that warming rates are tightly dependent on cooling rates due to the presence of small ice fractions inside vitrified cells. Consistent with this, Seki et al. [28] state that during the cooling process, small ice crystals form; consequently, these crystals could cause recrystallization during the warming process. Thus, faster cooling rates require faster warming rates to block recrystallization [33]. Seki et al. [28] showed that moderate cooling rates combined with high warming rates of $117,500{ }^{\circ} \mathrm{C} / \mathrm{min}$ can prevent recrystallization. An ultra-high warming rate of $10,000,000{ }^{\circ} \mathrm{C} / \mathrm{min}$ can be achieved by using a laser pulse, which is a novel method for ultra-rapid warming [45]. Although it is clear that rapid warming enables cells to pass through the recrystallization process, the concentration of CPAs needs to be taken into account. An inadequate CPA level can cause recrystallization during the warming process [29]. In agreement with this assertion, Seki et al. [28] contend that the short time (1-2 minutes) for which embryos are exposed to equilibration solution is not enough for permeable solutes to penetrate cells and replace water molecules (through dehydration). However, recent studies have indicated that if the rate of warming is extremely rapid cooling rates and use of cryoprotectants appear not to be as important to the survival of oocytes and embryos after vitrification as was previously thought [46-48].

\section{Cryoprotectants}

CPAs play a central role in protecting cells from cryo-damage. Permeating and non-permeable CPAs are commonly used in gamete and embryo cryopreservation. Permeating compounds can penetrate into cells and replace intracellular water; consequently, these cells rehydrate and are protected from ice crystal formation [49]. Non-electrolyte CPAs of low molecular weight enter cells with different rates of penetration [1]. Frequently used permeable CPAs include dimethyl sulfoxide ( $\left.\mathrm{Me}_{2} \mathrm{SO}\right)$, glycerol, ethylene glycol (EG), methanol and propylene glycol. According to Leibo [1], oocytes and embryos are equilibrated with CPAs at half the concentration of the final vitrification solution. For example, in Cryotop ${ }^{\varpi}$ - a typical vitrification - embryos are first exposed to an equilibration solution containing 7.5\% v/v EG and 7.5\% v/v Me $\mathrm{SO}$, then to a vitrification solution of 15\% v/v EG and $15 \% \mathrm{v} / \mathrm{v}$ $\mathrm{Me}_{2} \mathrm{SO}$. Permeability of cells is also dependent on type of CPA. Mazur et al. [30] found that glycerol does not permeate mouse oocytes. EG is more permeable to embryos than is glycerol, so EG is a preferred CPA in the field with a one-step dilution [50].

In order to avoid intracellular ice crystal formation [28] and to achieve vitrification [36], a high concentration of CPA is required; however, this may cause osmotic stress [51]. Vanderzwalmen et al. [52] contend that both a high intracellular and extracellular concentration of CPAs is necessary to minimize excessive swelling of embryos, which is a consequence of water entrance during the warming process. Thus, non-permeable CPAs such as the sugars sucrose and trehalose are used to lessen osmotic stress. Dobrinsky [53] considers that CPAs protect intracellular organelles during the cooling and warming processes; on the other hand, their toxic characteristics can cause osmotic injuries to the cells [22,54].

While Fahy [54] contends that CPA toxicity is a major drawback of cryopreservation by either slow freezing or vitrification, minimizing toxic effects makes this approach a more attractive proposition. It is a common perception that a high concentration of CPAs used in vitrification can be harmful to oocytes and embryos. Interestingly, some recent opposing views attempt to redress this perception. Vanderzwalmen et al. [52] argue that intracellular CPA levels in vitrified embryos are significantly lower than those in frozen embryos. In addition, provided that the warming rate reaches $1 \times 10^{7} \mathrm{C}$, even when the permeating CPA concentration in vitrification solution is reduced to one half [46], one third [47] or zero [48], survival rates of mouse oocytes and embryos after vitrification can attain close to $100 \%$. Jin and Mazur [48] maintain that the molarity of permeating CPAs penetrating into oocytes and embryos is not as important for their survival as the volume of cytoplasmic water that has been withdrawn from these cells before vitrification.

\section{Suitability of Both Slow Cooling and Vitrification for In Vivo-Derived Embryos}

In vivo embryos are generated in donors with assistance of exogenous gonadotropin hormones, and the majority of these embryos are frozen. Although slow freezing has become accepted practice for in vivo embryo cryopreservation, vitrification is the method of choice for storing in vivo embryos. Van Wagtendonk-de Leeuw et al. [55] report that vitrification and slow freezing resulted in similar conception rates in recipients. In North America 70\% of superovulated embryos are slow frozen, so this is the apparent method of choice for cryopreserving in vivo embryos when a programmable freezer is available [2]. Hasler [3] reports high overall pregnancy rates of recipients implanted with in vivo frozen embryos (56.1\%). Moreover, Lopatarova et al. [56] demonstrated a $48.8 \%$ conception rate for recipients implanted with frozen and biopsied in vivo embryos, while that for recipients of intact and frozen in vivo embryos was 50.7\%. While confirming that vitrification is sometimes recommended to cryopreserve in vivo embryos, Hasler [2] maintains that slow freezing and vitrification produce similar pregnancy rates among recipients. Since slow freezing has become a standard method for in vivo bovine embryo cryopreservation, further research on refining this technique may enable improved outcomes for embryo production [2,57]. 


\section{In Vitro-Fertilized Embryo Cryopreservation}

In contrast to in vivo embryo cryopreservation slow freezing may not be efficient for storage of IVP embryos. Seidel [13] recognizes the difficulty when cryopreserving ruminant in vitro-derived embryos. Table 1 summarizes a series of experimental studies which demonstrates that survival rates of IVP frozen bovine embryos after thawing and further culture in vitro are lower than those of vitrified embryos.

\begin{tabular}{|c|c|c|}
\hline Source & Slow freezing & Vitrification \\
\hline Mahmoudzadeh et al. [5] & 43.3 & 71.3 \\
\hline Enright et al. [6] & 0 & 17 \\
\hline Nedambale et al. [7] & 22 & 54 \\
\hline Mucci et al. [4] & 12 & 43 \\
\hline Yu et al. [8] & 24.7 & 34.9 \\
\hline
\end{tabular}

Table 1: Percentage hatching rates of bovine IVP blastocysts following slow freezing or vitrification (listed in chronological order of publication)

Enright et al. [6] reported that no in vitro embryos survived when recovered from frozen. Similarly, Nedambale et al. [7] observed that the number of cells damaged by fragmentation was higher in frozen embryos than in vitrified embryos. For goats, Begin et al.[58] and Al Yacoub et al. [59] reported that OPS vitrification was utilized successfully for in vitro-derived goat embryos.

Although vitrification appears to be preferable to slow freezing, further investigation is required [20]. While several data sets on pregnancy rates are available [7,24,35], the sample sizes in these studies were relatively small. For example, Gutnisky et al. [24] transferred vitrified embryos to recipients and obtained $46.8 \%$ conception rates. However, as the number of embryos used was modest $(n=96)$, this may not provide an accurate representation nor reflect the potential efficacy of the vitrification method. Also, a calving rate of only $34.3 \%$ does not represent a promising outcome under commercial conditions where the ultimate goal of an embryo transfer program is to achieve a high yield of healthy livestock. Previously, Enright et al. [6] reported that the abortion rate of IVP embryos in recipient cows is high and that due care should be taken with pregnancy interpretation.

\section{Challenges Facing Cryopreservation of IVP Bovine Embryos}

The cytoplasmic lipid content of oocytes facilitates mitochondrial activity essential to early development of embryos [60]. Additionally, Ferguson and Leese [61] showed that the triglyceride in bovine oocytes is a nutrient that provides energy for oocytes and embryos during the maturation and fertilization processes. The amount of lipid in in vitro-derived embryos that were cultured in a serum-free medium was similar to their in vivo-derived counterparts, but the lipid content of embryos cultured in the presence of serum was nearly twice as much as those originating from in vivo embryos [61].

Block et al. [62] indicated that cytoplasmic lipid droplets in embryos have no effect on their viability after cryopreservation; however, others have argued that the ability of cryopreserved embryos to survive is related to the presence of such lipid content $[13,63]$. Seidel [13] and Pryor et al. [11] note that there is a correlation between lipid droplets and embryo cryotolerance. Moreover, Cagnone and Sirard [63] maintain that the in vitro culture system affects the lipid content of embryos and influences their subsequent cryo-survival. Previously, Abe et al. [10] had found that the sensitivity of in vitro embryos to cryopreservation is due to the substantial accumulation of lipid droplets in their cytoplasm. Embryos cultured in serum-free medium contain mostly small lipid droplets $(<2 \mu \mathrm{m})$; in contrast, embryos cultured in serum-supplemented medium possess larger lipid droplets. Large droplets $(>62 \mu \mathrm{m})$ were often observed in morulae and blastocysts when cultured in media containing 5\% v/v calf serum [10]. This abnormal accumulation of lipid in the cytoplasm resulting from culture in the presence of serum is the main reason IVP embryos are considered unsuitable for cryopreservation [14]. Mucci et al. [4] point out that elevated levels of lipid droplets in embryos affect cellular repair after cryopreservation. Furthermore, Sudano et al. [12] contend that high concentrations of fetal calf serum in in vitro culture media lead to increased lipid accumulation in blastocysts, and apoptosis, but reduced expansion of blastocoels after vitrification. In sheep, it is also evident that addition of fetal calf serum to culture media increases lipid droplet abundance [64].

\section{Direct Transfer of Vitrified IVP Embryos}

Most vitrification carriers are not designed for direct transfer because they are poorly suited to use with a standard $0.25 \mathrm{~mL}$ plastic straw [65]. In addition, vitrification includes several steps in which embryos are placed into equilibrium and vitrification media; thus, warming involves multiple steps to eliminate CPAs. Simplification of the procedure by a single warming step is necessary to make possible the direct transfer of vitrified bovine embryos. It is evident that warming by single or multiple steps achieves similar results [9,51]. Vajta et al. [66] developed an in-straw dilution protocol in which a mini French straw is loaded with holding medium and $0.2 \mathrm{M}$ sucrose; however, this procedure requires skilled technicians [67]. Another drawback of this modification is the loss of embryos during warming [9]. Vieira et al. [67] state that warming in air for 3-5 seconds before an embryo is loaded possibly reduces the warming rate, which is critical to its survival [33]. Morato and Mogas [51] found that keeping the warming solution at $45^{\circ} \mathrm{C}$ facilitates a better survival rate of vitrified embryos than at $50{ }^{\circ} \mathrm{C}, 60{ }^{\circ} \mathrm{C}$ or $70{ }^{\circ} \mathrm{C}$. In accord, Caamano et al. [9] record that 41 ${ }^{\circ} \mathrm{C}$ is the most suitable temperature to warm vitrified embryos. Although reports of direct transfer have provided promising results $[9,51,67]$, embryo losses, evaluated through transfer, are limiting. 


\section{Modifications to In Vitro Culture Systems}

If, as the literature suggests, chilling sensitivity of in vitro-derived bovine embryos is related to the amount of lipid in the cytoplasm, adapting IVP conditions to reduce the lipid content appears necessary to increase cryo-tolerance. Serum has been shown to increase the amount of lipids; thus, embryologists have attempted to modify in vitro culture media by substituting serum-based media for chemical-defined ones. Seidel [13] reported that in vitro-fertilized embryos cultured in a chemical-defined medium without blood serum can be more cryotolerant than those cultured in bovine serum albumin (BSA) or a medium with added fetal calf serum (FCS). It is important to note that serum supplementation of in vitro culture media plays a crucial role in the development of oocytes and embryos because serum provides nutrients, vitamins, growth factors and antioxidants necessary for the healthy development of in vitro-fertilized embryos [63]. In contrast to previous reports, Castaneda et al. [68] claim that bovine oocytes with a high number of lipid droplets show greater developmental ability since lipid sources provide energy for functioning intracellular systems.

Studies on modifications to in vitro culture systems have sought to keep a sufficient concentration of FCS and to add redox regulator compounds such as phenazine ethosulfate to enhance the viability of embryos after cryopreservation. Sudano et al. [12] found that cytoplasmic lipid can be reduced by supplementation of $0.25 \% \mathrm{v} / \mathrm{v}$ FCS. Moreover, in combination with exposure of embryos at day four to phenazine ethosulfate, which restricts fatty acid synthesis, embryo quality and their viability after vitrification was improved. These authors also argue that the cryosurvival of embryos is dependent not only on lipid content but also apoptosis [12]. Consistent with this, Barcelo-Fimbres and Seidel [69] demonstrated that phenazine ethosulfate decreases lipid composition while serum increases lipid accumulation in bovine embryos, yielding expanded blastocysts with few cytoplasmic lipid droplets. It is also recommended that the in vitro culture medium is supplemented with other lipolytic substances. For example, Sanches et al. [25] showed that addition of forskolin to an in vitro culture medium on day five post in vitro fertilization leads to satisfactory pregnancy rates of recipients.

\section{Enhancement of Some Specific Features of Embryos}

Improving particular characteristics of in vitro-fertilized bovine embryos is another means to enhance their quality prior to cryopreservation. Appropriate exposure of gametes and embryos to sub-lethal hydrostatic pressure raises their intrinsic developmental competence to overcome extrinsic stressors such as the cooling process [70,71]. An enhancement in cell performance is possibly due to high hydrostatic pressure (HHP) causing a minimum stressful condition under which cells can produce and accumulate heat shock proteins [72,73]. For example, Pribenszky et al. [72] found that HHP treatment of pig oocytes before Cryotop ${ }^{\oplus}$ vitrification increased rates of cleavage and blastocyst formation. Similarly, Filho et al. [26], pretreating bovine blastocysts with HHP, noted that re-expansion and hatching rates of treated embryos are significantly higher than those of untreated embryos. However, pregnancy rates were not reported. Evidently, this promising approach to promoting oocyte and embryo survival merits further investigation.

\section{Sophisticated Techniques to Improve Cryopreservation Outcomes}

Micro-manipulation of in vitro-fertilized embryos prior to vitrification may increase the survival of treated embryos after cryopreservation. For instance, sufficient dehydration of blastocysts before vitrification, achieved by artificially collapsing the blastocoel, improves their implantation potential [74]. Using this technique, Min et al. [27] observed increased survival of in vitro bovine embryos and cloned embryos post-warming and a further 24 hours culture. Survival rates of in vitro embryos following blastocoel forced treatments were significantly higher than in control groups (81.9\% versus 69.8\%). However, this protocol is complicated by time requirements and the necessity of the embryologist to be highly proficient. Unlike Min et al. [27], Pryor et al. [11] used a laser-assisted hatching method whereby a laser micro-beam drills a hole in the zona pellucida through which lipid droplets can escape from the cytoplasm. However, this delipidation technique appears to be technically complex.

\section{Embryo Biosafety}

Biosafety is a primary consideration for the exportation and importation of bovine embryos between countries. Hence, it is important to recognize the potential for pathogen transmission following transfer of cryopreserved gametes and embryos. Vitrification has two distinct systems, closed and open, which present different risks. Similar to slow freezing in straws, a closed vitrification system has a reduced risk of microbial contamination compared to methods requiring direct contact of embryos with liquid nitrogen. In theory at least, direct contact of the vitrification solution with liquid nitrogen may result in contamination [17]. Morris [75] argues that it is a false assumption to think that liquid nitrogen is sterile and points to the possibility that contamination could originate in cryopreserved samples. Furthermore, Mirabet et al. [76] maintain that various viruses are able to survive at subzero temperatures without CPA requirements and cryopreservation methods. Bielanski et al. [77] tested the feasibility of contamination from liquid nitrogen of three species of virus - bovine immunodeficiency virus (BIV), bovine viral diarrhoea virus (BVDV) and bovine herpes virus $(\mathrm{BHV})$. While unsealed carriers were either positive or negative for these viruses, sealed ones were all negative. Although identifying 32 bacterial and one fungal species from liquid nitrogen storing semen and embryos over a 35 year period, Bielanski et al. [78] did not detect BVDV and BHV in clean semen and embryo straws stored with infected straws. Nevertheless, it is not straightforward to keep samples clean if contained alongside unclean ones in the same liquid nitrogen tank. It is important to note that although cryopreservation can reduce the percentage of embryos infected by BVDV and BHV, this process may not totally remove the risk of infectious pathogens [79]. 
In spite of low microbial contamination risks relating to contact of samples with liquid nitrogen, it is worth considering these potential hazards [80]. A closed system may not attain high enough cooling rates, but an open system does; hence, compromising technical and sanitary issues is rather difficult. Yu et al. [8] modified OPS to closed pulled straw (CPS) to eliminate the chance of contamination. Although these authors claim CPS maintains the advantages of OPS, the survival rates of embryos vitrified by CPS were relatively low.

\section{Conclusion}

Intracellular formation of ice crystals during cooling and warming processes is the principal cause of cryo-damage to cells. Cryopreservation by slow freezing and vitrification are aimed to minimize or, ideally, to totally eliminate this accumulation. CPAs are indispensable for protection from chilling but they are possibly toxic to the cell. While both slow freezing and vitrification are efficient methods of cryopreserving in vivo-derived bovine embryos, slow freezing may not be suitable for in vitro-fertilized embryos. This may be due to the fact that in vitro-fertilized bovine embryos contain more cytoplasmic lipid droplets than do their in vivo counterparts, although improved culture conditions may ameliorate this. Considerable research has indicated the advantages of vitrification to become the method of choice for storing in vitro-derived embryos. In order to further improve outcomes a greater understanding is required of cooling and warming, the role of CPAs, modifications to in vitro culture media and to treatment of cells prior to cryopreservation. Moreover, biosafety of embryos is an important factor that warrants investigation. Although vitrification is apparently superior to slow freezing to cryopreserve in vitro-fertilized bovine embryos, non-standardization of techniques and an inconsistency of results between laboratories may hamper widespread adoption of vitrification. Hence, it is necessary to identify the most efficient way to generate IVP blastocysts with a reduced lipid content as well as optimizing vitrification methods for in vitro-fertilized bovine embryos. In turn, this will enhance theoretical understanding of bovine cryobiology while, from a practical perspective, facilitating increased use of in vitro embryos in the field.

\section{References}

1. Leibo SP (2008) Cryopreservation of oocytes and embryos: optimization by theoretical versus empirical analysis. Theriogenology 69: 37-47.

2. Hasler JF (2014) Forty years of embryo transfer in cattle: a review focusing on the journal Theriogenology, the growth of the industry in North America, and personal reminisces. Theriogenology 81: 152-69.

3. Hasler JF (2001) Factors affecting frozen and fresh embryo transfer pregnancy rates in cattle. Theriogenology 56: 1401-15.

4. Mucci N, Aller J, Kaiser GG, Hozbor F, Cabodevila J, et al. (2006) Effect of estrous cow serum during bovine embryo culture on blastocyst development and cryotolerance after slow freezing or vitrification. Theriogenology 65: 1551-62.

5. Mahmoudzadeh AR, Van Soom A, Ysebaert MT, de Kruif A (1994) Comparison of two-step vitrification versus controlled freezing on survival of in vitro produced cattle embryos. Theriogenology 42: 1389-97.

6. Enright BP, Lonergan P, Dinnyes A, Fair T, Ward FA, et al. (2000) Culture of in vitro produced bovine zygotes in vitro vs in vivo: implications for early embryo development and quality. Theriogenology 54: 659-73.

7. Nedambale TL, Dinnyes A, Groen W, Dobrinsky JR, Tian XC, et al. (2004) Comparison of in vitro fertilized bovine embryos cultured in KSOM or SOF and cryopreserved by slow freezing or vitrification. Theriogenology 62: 437-49.

8. Yu XL, Deng W, Liu FJ, Li YH, Li XX, et al. (2010) Closed pulled straw vitrification of in vitro-produced and in vivo-produced bovine embryos. Theriogenology 73: 474-9.

9. Caamano JN, Gomez E, Trigal B, Munoz M, Carrocera S, et al. (2015) Survival of vitrified in vitro-produced bovine embryos after a one-step warming in-straw cryoprotectant dilution procedure. Theriogenology 83: 881-90.

10. Abe H, Yamashita S, Itoh T, Satoh T, Hoshi H (1999) Histochemical and ultrastructural evaluations of cytoplasmic lipid droplets in bovine embryos cultured in serum-free and serum-containing media. Theriogenology 51: 232.

11. Pryor JH, Looney CR, Romo S, Kraemer DC, Long CR (2011) Cryopreservation of in vitro produced bovine embryos: effects of lipid segregation and post-thaw laser assisted hatching. Theriogenology 75: 24-33.

12. Sudano MJ, Paschoal DM, Rascado TS, Magalhaes LCO, Cromoco LF, et al. (2011) Lipid content and apoptosis of in vitro-produced bovine embryos as determinants of susceptibility to vitrification. Theriogenology 75: 1211-20.

13. Seidel GE (2006) Modifying oocytes and embryos to improve their cryopreservation. Theriogenology 65: 228-35.

14. Pereira RM, Carvalhais I, Pimenta J, Baptista MC, Vasques MI, et al. (2008) Biopsied and vitrified bovine embryos viability is improved by trans10 and cis 12 conjugated linoleic acid supplementation during in vitro embryo culture. Anim Reprod Sci 106: 322-32.

15. van Wagtendonk-de LeeuwAM (2005) Ovum pick up and in vitro production in the bovine after use in several generations: a 2005 status. Theriogenology 65 : 914-25.

16. Pontes JHV, MeloSterza FA, Basso AC, Ferreira CR, Sanches BV, et al. (2011) Ovum pick up, in vitro embryo production, and pregnancy rates from a large-scale commercial program using Nelore cattle (Bos indicus) donors. Theriogenology 75: 1640-6.

17. Vajta G, Rienzi L, Ubaldi FM (2015) Open versus closed systems for vitrification of human oocytes and embryos. Reprod BioMed Online 30: 325-33.

18. Taylor-Robinson AW, Walton S, Swain DL, Walsh KB, Vajta G (2014) The potential for modification in cloning and vitrification technology to enhance genetic progress in beef cattle in Northern Australia. Anim Reprod Sci 148: 91-6.

19. Rios GL, Mucci NC, Kaiser GG, Alberio RH (2010) Effect of container, vitrification volume and warming solution on cryosurvival of in vitro-produced bovine embryos. Anim Reprod Sci 118: 19-24.

20. Sinha S (2009) Role of cryopreservation in assisted reproductive technology (ART). Apollo Medicine 6: 212-21.

21. Gomez E, Rodriguez A, Munoz M, Caamano JN, Hidalgo CO, et al. (2008) Serum free embryo culture medium improves in vitro survival of bovine blastocysts to vitrification. Theriogenology 69: 1013-21. 
22. Wu G, Jia B, Mo X, Liu C, Fu X, et al. (2013) Nuclear maturation and embryo development of porcine oocytes vitrified by cryotop: effect of different stages of in vitro maturation. Cryobiology 67: 95-101.

23. Vajta G, Holm P, Kuwayama M, Booth PJ, Jacobsen H, et al. (1998) Open pulled straw (OPS) vitrification:a new way to reduce cryoinjuries of bovine ova and embryos. Mol Reprod Dev 51: 53-8.

24. Gutnisky C, Alvarez GM, Cetica PD, Dalvit GC (2013) Evaluation of the Cryotech Vitrification Kit for bovine embryos. Cryobiology 67: $391-3$.

25. Sanches BV, Marinho LSR, Filho BDO, Pontes JHF, Basso AC, et al. (2013) Cryosurvival and pregnancy rates after exposure of IVF-derived Bos indicus embryos to forkolin before vitrification. Theriogenology 80: 372-7.

26. Siqueira Filho E, Caixeta ES, Pribenszky C, Molnar M, Horvath A, et al. (2011) Vitrification of bovine blastocysts pretreated with sublethal hydrostatic pressure stress: evaluation of post-thaw in vitro development and gene expression. Reprod Fertil Dev 23: 585-90.

27. Min SH, Lee E, Son HH, Yeon JY, Koo DB (2013) Forced collapse of the blastocoel enhances survival of cryotop vitrified bovine hatching/hatched blastocysts derived from in vitro fertilization and somatic cell nuclear transfer. Cryobiology 66: 195-9.

28. Seki S, Jin B, Mazur P (2014) Extreme rapid warming yields high functional survivals of vitrified 8-cell mouse embryos even when suspended in a half-strength vitrification solution and cooled at moderate rates to $-196^{\circ} \mathrm{C}$. Cryobiology 68: 71-8.

29. Jin B, Kusanagi K, Ueda M, Seki S, Valdez DM, et al. (2008) Formation of extracellular and intracellular ice during warming of vitrified mouse morulae and its effect on embryo survival. Cryobiology 56: 233-40.

30. Mazur P, Seki S, Pinn IL, Kleinhans FW, Edashige K (2005) Extra- and intracellular ice formation in mouse oocytes. Cryobiology 51: 29-53.

31. Mazur P, Pinn IL, Kleinhans FW (2007) Intracellular ice formation in mouse oocytes subjected to interrupted rapid cooling. Cryobiology 55: 158-66.

32. Lee KH, Sun JC, Chuang C, Guo SF, Tu CF, et al. (2013) An efficient and mass reproducible method for vitrifying mouse embryos on a paper in cryotubes. Cryobiology 66: 311-7.

33. Mazur P, Seki S (2011) Survival of mouse oocytes after being cooled in a vitrification solution to $-196{ }^{\circ} \mathrm{C}$ at $95^{\circ} \mathrm{C}$ to $70,000{ }^{\circ} \mathrm{C} / \mathrm{min}$ and warmed at $610^{\circ}$ to $118,000{ }^{\circ} \mathrm{C} / \mathrm{min}$ : a new paradigm for cryopreservation by vitrification. Cryobiology 62: 1-7.

34. Kleinhans FW, Mazur P (2009) Determination of the water permeability (Lp) of mouse oocytes at $-25^{\circ} \mathrm{C}$ and its activation energy at subzero temperatures. Cryobiology 58: 215-24.

35. Kim YM, Uhm SJ, Gupta MK, Yang JS, Lim JG, et al. (2012) Successful vitrification of bovine blastocysts on paper container. Theriogenology 78: 1085-93.

36. Lawson A, Mukherjee IN, Sambanis A (2012) Mathematical modeling of cryoprotectant addition of engineered or natural tissues. Cryobiology 64: 1-11.

37. Kobayashi A, Kirschvink JL (2014) A ferromagnetic model for the action of electric and magnetic fields in cryopreservation. Cryobiology 68: 163-5.

38. Palasz AT, Gustafsson H, Rodriguez-Martinez H, Gusta L, Larsson B, et al. (1997) Vitrification of bovine IVF blastocysts in an ethylene glycol/sucrose and heatstable plant-extracted proteins. Theriogenology 47: 865-79.

39. Kuwayama M (2007) Highly efficient vitrification for cryopreservation of human oocytes and embryos: Cryotop method. Theriogenology 67: 73-80.

40. Matsunari H, Maehara M, Nakano K, Ikezawa Y, Hagiwara Y, et al. (2012) Hollow fiber vitrification: a novel method for vitrifying multiple embryos in a single device. J Reprod Dev 58: 599-608.

41. Paredes E, Mazur P (2013) The survival of mouse oocytes shows little or no correlation with the vitrification or freezing of the external medium, but the ability of the medium to vitrify is affected by its solute concentration and by the cooling rate. Cryobiology 67: 386-90.

42. Zhou XL, Naib AAl, Sun DW, Lonergan P (2010) Bovine oocyte vitrification using the Cryotop method: effect of cumulus cells and vitrification protocol on survival and subsequent development. Cryobiology 61: 66-72.

43. Sansinena M, Santos MV, Taminelli G, Zaritky N (2014) Implications of storage and handling conditions on glass transition and potential devitrification of oocytes and embryos. Theriogenology 82: 373-8.

44. Hopkins JB, Badeau R, Warkentin M, Thorne RE (2012) Effect of common cryoprotectants on critical warming rates and ice formation in aqueous solutions. Cryobiology 65: 169-78.

45. Kleinhans FW, Mazur P (2015) Physical parameters and methodological details in using IR laser pulses to warm frozen or vitrified cells ultra-rapidly. Cryobiology 70: 195-203.

46. Seki S, Mazur P (2012) Ultra-rapid warming yields high survival of mouse oocytes cooled to - $196^{\circ} \mathrm{C}$ in dilute vitrification solutions. PLoS One 7: e36058.

47. Jin B, Kleinhans FW, Mazur P (2014) Survivals of mouse oocytes approach $100 \%$ after vitrification in 3-fold diluted media and ultra-rapid warming by an IR laser pulse. Cryobiology 68: 419-30.

48. Jin B, Mazur, P (2015) High survival of mouse oocytes/embryos after vitrification without permeating cryoprotectants followed by ultra-rapid warming with an IR laser pulse. Nature Sci Rep 5: 9271.

49. Palasz AT, Mapletoft RJ (1996) Cryopreservation of mammalian embryos and oocytes: recent advances. Biotech Adv 14: 127-49.

50. Dochi O, Yamamoto Y, Saga H, Yoshiba N, Kano N, et al. (1998) Direct transfer of bovine embryos frozen-thawed in the presence of propylene glycol or ethylene glycol under on-farm conditions in an integrated embryo transfer program. Theriogenology 49: 1051-8.

51. Morato R, Mogas T (2014) New device for the vitrification and in-straw warming of in vitro produced embryos. Cryobiology 68: $288-93$.

52. Vanderzwalmen P, Grobet L, Zech NH, Puissant F, Ectors F (2012) Lower intracellular concentration of cryoprotectant (ICCP) after vitrification (VIT) than slow freezing (SF) despite exposure to high concentration of cryoprotectant solutions (CPS). Fertil Sterility 98: 49.

53. Dobrinsky JR (2002) Advancements in cryopreservation of domestic animal embryos. Theriogenology 57: $285-302$.

54. Fahy GM (2010) Cryoprotectant toxicity neutralization. Cryobiology 60: S45-53.

55. van Wagtendonk-de Leeuw AM, den Daas JHG, Rall WF (1997) Field trial to compare pregnancy rates of bovine embryo cryopreservation methods: vitrification and one-step dilution versus slow freezing and three-step dilution. Theriogenology 48: 1071-84.

56. Lopatarova M, Cech S, Krontorad P, Holy L, Lalova H, et al. (2010) Conception rate after sex determination and cryopreservation of D7 bovine embryos. Vet Med (Praha) 55: 10-18. 
57. Vajta G, Nagy ZP (2006) Are programmable freezers still needed in the embryo laboratory? Review on vitrification. Reprod BioMed Online 12: 779-96.

58. Begin I, Bhatia B, Baldassarre H, Dinnyes A, Keefer CL (2003) Cryopreservation of goat oocytes and in vivo derived 2- to 4-cell embryos using the cryoloop (CLV) and solid-surface vitrification (SSV) methods. Theriogenology 59: 1839-50.

59. Al Yacoub AN1, Gauly M, Holtz W (2010) Open pulled straw vitrification of goat embryos at various stages of development. Theriogenology 73: 1018-23.

60. Jeong WJ, Cho SJ, Lee HS, Deb GK, Lee YS, et al. (2009) Effect of cytoplasmic lipid content on in vitro developmental efficiency of bovine IVP embryos. Theriogenology 72: 584-9.

61. Ferguson EM, Leese HJ (1999) Triglyceride content of bovine oocytes and early embryos. J Reprod Fertil 116: $373-8$.

62. Block, J, Bonilla, L \& Hansen, PJ (2010) Efficacy of in vitro embryo transfer in lactating dairy cows using fresh or vitrified embryos produced in a novel embryo culture medium. J Dairy Sci 93: 5234-42.

63. Cagnone G, Sirard MA (2014) The impact of exposure to serum lipids during in vitro culture on the transcriptome of bovine blastocysts. Theriogenology 81 : 712-22.

64. Reis A, McCallum GJ, McEvoy TG (2005) Accumulation and distribution of neutral lipid droplets is non-uniform in ovine blastocysts produced in vitro in either the presence or absence of serum. Reprod Fertil Dev 17: 815-23.

65. Ha AN, Lee SR, Jeon JS, Park HS, Lee SH, et al. (2014) Development of a modified straw method for vitrification of in vitro-produced bovine blastocysts and various genes expression in between the methods. Cryobiology 68: 57-64.

66. Vajta G, Murphy CN, Machaty Z, Prather RS, Greve T, et al. (1999) In-straw dilution of bovine blastocysts after vitrification with the open-pulled straw method. Vet Rec 144: 180-1.

67. Vieira AD, Forell F, Feltrin C, Rodrigues JL (2007) In-straw cryoprotectant dilution of IVP bovine blastocysts vitrified in hand-pulled glass micropipettes. Anim Reprod Sci 99: 377-83.

68. Castaneda CA, Kaye P, Pantaleon M, Phillips N, Norman S, et al. (2013) Lipid content, active mitochondria and brilliant cresyl blue staining in bovine oocytes. Theriogenology 79: 417-22.

69. Barcelo-Fimbres M, Seidel GE (2011) Cross-validation of techniques for measuring lipid content of bovine oocytes and blastocysts. Theriogenology 75: 434-44.

70. Pribenszky C, Vajta G, Molnar M, Du Y, Lin L, et al. (2010) Stress for stress tolerance? A fundamentally new approach in mammalian embryology. Biol Reprod 83: 690-7.

71. Pribenszky C, Vajta G (2011) Cells under pressure: how sublethal hydrostatic pressure treatment increases gametes' and embryos' performance. Reprod Fertil Dev 23: 48-55.

72. Pribenszky CS, Du Y, Molnar M, Harnos A, Vajta G (2008) Increased stress tolerance of matured pig oocytes after high hydrostatic pressure treatment. Anim Reprod Sci 106: 200-7.

73. Diez C, Munoz M, Caamano JN, Gomez E (2012) Cryopreservation of the bovine oocyte: current status and perspectives. Reprod Domest Anim 47: 76-83.

74. Pelts EJ, Mathews JM, Sanchez SR, Rapisards J, Ledere K (2012) Does artificial collapse of human day 6 blastocysts prior to the cooling steps of vitrification improve their probability of increased outcome. Fertil Sterility 98: 52.

75. Morris GJ (2005) The origin, ultrastructure, and microbiology of the sediment accumulating in liquid nitrogen storage vessels. Cryobiology 50: 231-8.

76. Mirabet V, Alvarez M, Solves P, Ocete D, Gimeno C (2012) Use of liquid nitrogen during storage in a cell and tissue bank: contamination risk and effect on the detectability of potential viral contaminants. Cryobiology 64: 121-3.

77. Bielanski A, Nadin-Davis S, Sapp T, Lutze-Wallace C (2000) Viral contamination of embryos cryopreserved in liquid nitrogen. Cryobiology 40: 110-6.

78. Bielanski A, Bergeron H, Lau PCK, Devenish J (2003) Microbial contamination of embryos and semen during long term banking in liquid nitrogen. Cryobiology 46: 146-52.

79. Bielanski A, Lalonde A (2009) Effect of cryopreservation by slow cooling and vitrification on viral contamination of IVF embryo experimentally exposed to bovine viral diarrhea virus and bovine herpes virus-1. Theriogenology 72: 919-25.

80. Vajta G, Kuwayama M (2006) Improving cryopreservation system. Theriogenology 65: 236-44.

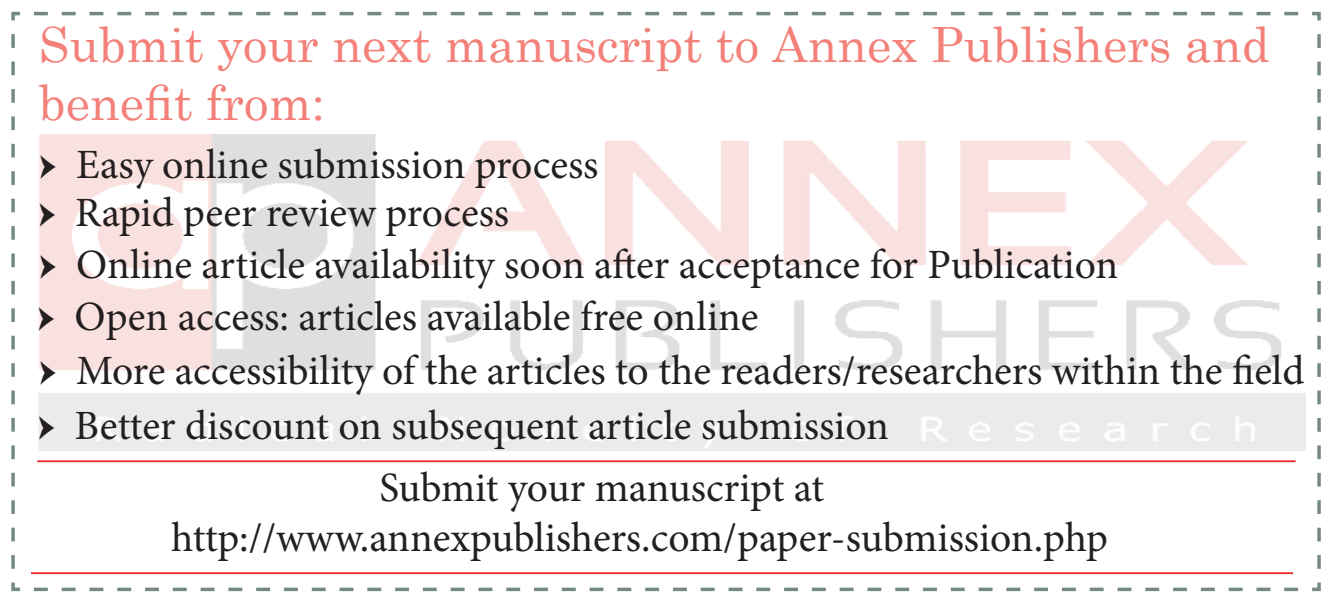

